\title{
Suppression of low-temperature ferromagnetic phase in ultrathin FeRh films
}

\author{
G. C. Han, ${ }^{1}$ J. J. Qiu, ${ }^{1}$ Q. J. Yap, ${ }^{1}$ P. Luo, ${ }^{1}$ T. Kanbe, ${ }^{2}$ T. Shige, ${ }^{2}$ D. E. Laughlin, ${ }^{3}$ \\ and J.-G. Zhu ${ }^{3}$ \\ ${ }^{1}$ Data Storage Institute, $A^{*}$ STAR, Republic of Singapore \\ ${ }^{2}$ Showa Denko K.K, 13-9, Shiba daimon 1-Chome, Minato-ku, Tokyo 105-8518, Japan \\ ${ }^{3}$ Data Storage Systems Center, Carnegie Mellon University, Pittsburgh, Pennsylvania 15213, USA
}

(Received 7 January 2013; accepted 11 March 2013; published online 27 March 2013)

\begin{abstract}
Highly ordered B2 FeRh films with sharp magnetic transitions from the antiferromagnetic (AF) to ferromagnetic (FM) states were prepared on thermally oxidized Si wafers with thicknesses as low as $10 \mathrm{~nm}$. It is found that the transition temperature increases as the thickness decreases from $80 \mathrm{~nm}$ to $15 \mathrm{~nm}$, and then decreases from $15 \mathrm{~nm}$ to $10 \mathrm{~nm}$. While the ratio of the residual magnetization to the maximum magnetization keeps nearly unchanged for the film thickness of $15 \mathrm{~nm}$ and larger, it increases significantly when the thickness is reduced to $10 \mathrm{~nm}$. This residual magnetization was suppressed by slightly increasing the Rh atomic content in $10 \mathrm{~nm}$ thick FeRh films. Low-pressure deposition is found to play an important role in the stabilization of the AF phase. By depositing FeRh films at an extremely low pressure of $0.057 \mathrm{~Pa}$, a residual magnetization as small as $13.5 \mathrm{emu} / \mathrm{cc}$ at $100 \mathrm{~K}$ was observed for a film with a nominal thickness of $10 \mathrm{~nm}$ deposited on Si wafer. This value was further reduced to $6 \mathrm{emu} / \mathrm{cc}$ when the film is deposited on $\mathrm{MgO}$ substrates due to much improved FeRh crystallinity. These results are in close agreement with theoretical predictions on defect and interface induced FM stabilization. (C) 2013 American Institute of Physics. [http://dx.doi.org/10.1063/1.4798275]
\end{abstract}

\section{INTRODUCTION}

B2 ordered FeRh alloys show a unique magnetic transition from the antiferromagnetic (AF) state at low temperature (LT) to the ferromagnetic (FM) state at elevated temperatures. This property has attracted increased interest in FeRh thin films due to its potential applications in magnetic random access memory (MRAM) ${ }^{1,2}$ and magnetic recording media. ${ }^{3,4}$ The main challenge for these applications is to prepare ultrathin FeRh films without a residual FM phase at LT and also that display a sharp transition to the FM state at elevated temperatures, and vice versa. As discussed in Ref. 3, FeRh layer with a thickness of $10 \mathrm{~nm}$ or less could provide sufficient exchange coupling strength between the storage layer and the assist layer to reduce switching field significantly without affecting the thermal stability of the storage layer at ambient temperature. However, a sharp AFFM transition of FeRh film was only achieved in films with a thickness of $14 \mathrm{~nm}$ and above. ${ }^{5}$ When the thickness was further reduced to $10 \mathrm{~nm}$, a large residual magnetization was observed along with a broad transition. ${ }^{6}$ Furthermore, the above mentioned films were grown on $\mathrm{MgO}$ (001) substrates, which cannot be utilized in practical applications. Some groups $^{7,8}$ have also reported the growth of FeRh films on amorphous substrates like glass and thermally oxidized $\mathrm{Si}$ substrates. However, a sharp transition was only achieved for thick films $(\sim 150 \mathrm{~nm})$ using these substrates. The origin of the residual FM phase in the ultrathin film remains unclear. Fan et al. ${ }^{9}$ ascribed the residual magnetization to the FM phase existing in a region within $6-8 \mathrm{~nm}$ of the FeRh near the top and/or bottom interfaces. Based on ab initio calculations, Lounis et al. ${ }^{10}$ reported that a FM state is stable up to 9 atomic layers in an Rh-terminated FeRh film. In addition, the effects on the surface of the film accompanying the
FeRh magnetostructural transition have been studied in Ref. 11, which shows that the surface strain relief fosters nucleation of the ferromagnetic phase.

In order to reduce the residual magnetization and understand its origin, systematic studies were performed on the magnetic properties of FeRh ultrathin $(\sim 10 \mathrm{~nm})$ films grown on $\mathrm{MgO}(001)$ substrates and $\mathrm{Si}(001)$ substrates (with $1 \mu \mathrm{m}$ thermal oxide). In comparison with the typical residual magnetization reported in literature (about $250 \mathrm{emu} / \mathrm{cc}$ ), ${ }^{6}$ the residual magnetization in this work was significantly reduced to $6 \mathrm{emu} / \mathrm{cc}$ through the fine tuning of the film composition and depositing the films at low pressure on $\mathrm{MgO}$ substrates.

\section{EXPERIMENTS}

FeRh thin films were grown on thermally oxidized $\mathrm{Si}$ (100) wafers by the co-sputtering of pure Fe and Rh targets in an ultrahigh vacuum sputtering system with a base pressure of $<5 \times 10^{-6} \mathrm{~Pa}$. A typical layer structure is $\mathrm{Si} / \mathrm{SiO} / \mathrm{MgO}(10) /$ $\mathrm{FeRh}(10) / \mathrm{Ru}(5)$, where the digital numbers indicate thicknesses of each layer in nanometers. The $\mathrm{MgO}$ seed layers and the $\mathrm{Ru}$ capping layers were both deposited at room temperature, while the FeRh films were deposited at a pressure of $0.059 \mathrm{~Pa}$ (unless specified otherwise) and a temperature of $650{ }^{\circ} \mathrm{C}$. The film thicknesses were controlled by adjusting the deposition time with deposition rates obtained from the measured thicknesses and known deposition times of reference films which were deposited at room temperature. The crystalline structures of the films were analyzed by X-ray diffraction (XRD). X-ray photoelectron spectroscopy (XPS) was used to determine the film composition. Magnetic properties such as the temperature dependence of the magnetization and hysteresis loops were measured using a superconducting quantum interference device (SQUID) with a maximum field and a 


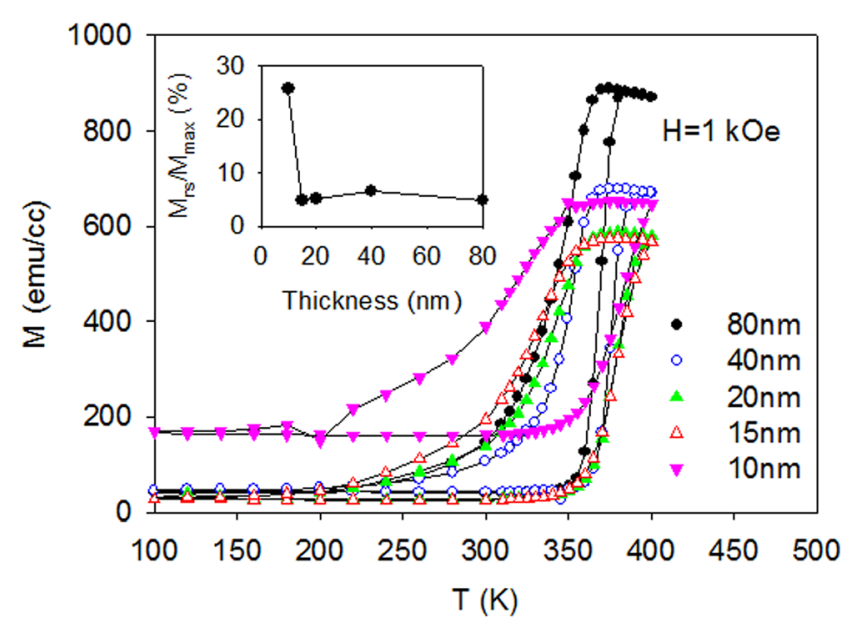

FIG. 1. Temperature dependence of magnetization measured at an applied field of $1 \mathrm{kOe}$ as a function of film thickness. The inset gives the ratio of the residual magnetization $\left(\mathrm{M}_{\min }\right)$ in the AF state to the maximum magnetization $\left(\mathrm{M}_{\max }\right)$ achieved in the FM state for various thicknesses.

highest temperature of $70 \mathrm{kOe}$ and $400 \mathrm{~K}$, respectively. The composition was considered optimized when the sputtering powers were fixed at $80 \mathrm{~W}$ and $87.5 \mathrm{~W}$ for Fe and Rh targets, respectively, to produce a smallest residual magnetization at $100 \mathrm{~K}$ for a $80 \mathrm{~nm}$ thick FeRh film.

\section{RESULTS AND DISCUSSION}

Fig. 1 shows the temperature dependence of the magnetization (M-T) with an applied field of $\mathrm{H}=1 \mathrm{kOe}$ for various thicknesses of FeRh layers. All these films clearly show an AF-FM phase transition. As the thickness decreases, the maximum magnetization achieved at $400 \mathrm{~K}$ after heating decreases and the transition hysteresis becomes broader, which is consistent with that reported in the literature. ${ }^{6}$ It is noted that although the hysteresis of the transition temperatures defined by the maximum derivative of the M-T curves for the $80 \mathrm{~nm}$ thick film is about $20 \mathrm{~K}$, similar to that reported before, ${ }^{5,6}$ there is a long tail for the FM-AF transition down to a temperature below $300 \mathrm{~K}$. This broadening of the FMAF transition could be attributed to the composition fluctuations in the film as well as the poorer crystalline orientations of the $\mathrm{MgO}$ buffer layers grown on the oxidized Si wafers as compared to $\mathrm{MgO}(001)$ substrates. Similar to that reported by Suzuki et al., ${ }^{6}$ the AF-FM transition temperature increases slightly as the film thickness decreases from $80 \mathrm{~nm}$ to $15 \mathrm{~nm}$ as observed in Fig. 1. This could be ascribed to the stress induced by the lattice-mismatch at the interface between $\mathrm{FeRh}$ and $\mathrm{MgO}$, analogous to the observation in high-pressure experiments on bulk FeRh. ${ }^{12,13}$ However, one can see the transition temperature is shifted to the lower temperature when the thickness is reduced from $15 \mathrm{~nm}$ to $10 \mathrm{~nm}$, which will be discussed later. As shown in the inset of Fig. 1 , it is found that the ratio of the residual magnetization $\left(\mathrm{M}_{\mathrm{rs}}\right)$ in the AF state to the maximum magnetization $\left(\mathrm{M}_{\max }\right)$ achieved in the FM state remains nearly unchanged at about $4.5 \%$ as the thickness decreases from $80 \mathrm{~nm}$ to $15 \mathrm{~nm}$. This result implies that this residual magnetization is not from the interface, but from the composition fluctuations throughout the film. When the thickness was further reduced from $15 \mathrm{~nm}$ to $10 \mathrm{~nm}$, the residual magnetization increases significantly, together with an increase in the total magnetization achieved at the FM state. The increase of the residual magnetization at the ultrathin thickness has been ascribed to the surface/interface effect due to the broken symmetry which contracts the $4 \mathrm{~d}$ band of the $\mathrm{Rh}$ atoms, increasing the density of states at the Fermi level [Ref. 10]. However, since the increase in residual magnetization is accompanied by an increase in the total magnetization at the FM state and the film thickness is much larger than that for the FM stabilization, we think the residual FM phase might arise from the formation of FM $\alpha^{\prime}$ phase due to the composition fluctuations over the film.

In order to reduce the FM $\alpha^{\prime}$ phase in the ultrathin FeRh film, the composition of the film was further tuned at a film thickness of $10 \mathrm{~nm}$. To do this, the sputtering power of the Fe target was fixed at $80 \mathrm{~W}$, while Rh sputtering power $\left(\mathrm{P}_{\mathrm{Rh}}\right)$ was increased gradually. As shown in Fig. 2, a slight increase of the Rh content reduces the residual magnetization significantly, which is most likely due to the suppression of the $\alpha^{\prime}$ phase or the transformation from the FM $\alpha^{\prime}$ phase to the AF $\alpha^{\prime \prime}$ phase. A small residual magnetization of about $18 \mathrm{emu} / \mathrm{cc}$ was obtained when $\mathrm{P}_{\mathrm{Rh}}=92.5 \mathrm{~W}$ and $95 \mathrm{~W}$. In contrast, Suzuki et al. ${ }^{6}$ reported a residual magnetization of $250 \mathrm{emu} /$ cc for a $10 \mathrm{~nm}$ thick FeRh film. As reported by van Driel et al. ${ }^{14} \mathrm{Rh}$ richer FeRh films have higher transition temperature and lower saturation magnetization. When the $\mathrm{Rh}$ content is further increased to $\mathrm{P}_{\mathrm{Rh}}=97 \mathrm{~W}$, as shown by filled downward triangles in Fig. 2, the residual magnetization increases along with a reduced magnetization after the AFFM transition occurs at about $400 \mathrm{~K}$. As shown in Fig. 3, XRD results show no sign of the $\gamma$ FeRh phase in the films as the sputtering power of $\mathrm{Rh}$ increases from $87.5 \mathrm{~W}$ to $97 \mathrm{~W}$. The background difference in XRD measurements for the sample deposited at $92.5 \mathrm{~W}$ resulted from the different alignment of XRD during $\theta-2 \theta$ scans. This result suggests that the thinner film needs more $\mathrm{Rh}$ to stabilize the AF phase than the thicker films. The additional $\mathrm{Rh}$ does not promote the formation of $\gamma \mathrm{FeRh}$ phase, but most likely forming $\mathrm{Rh}$ terminated grains. The AF stabilization in the Rh-terminated grains seems to contradict the results based on the ab initio

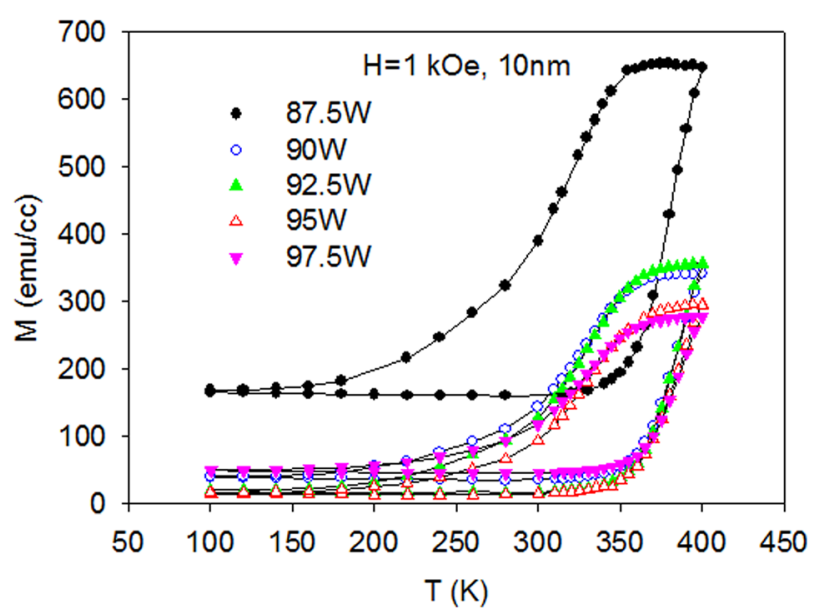

FIG. 2. M-T curves of $10 \mathrm{~nm}$ thick films deposited with different sputtering powers for $\mathrm{Rh}$ target for the fine tuning of $\mathrm{Rh}$ content in the films. 


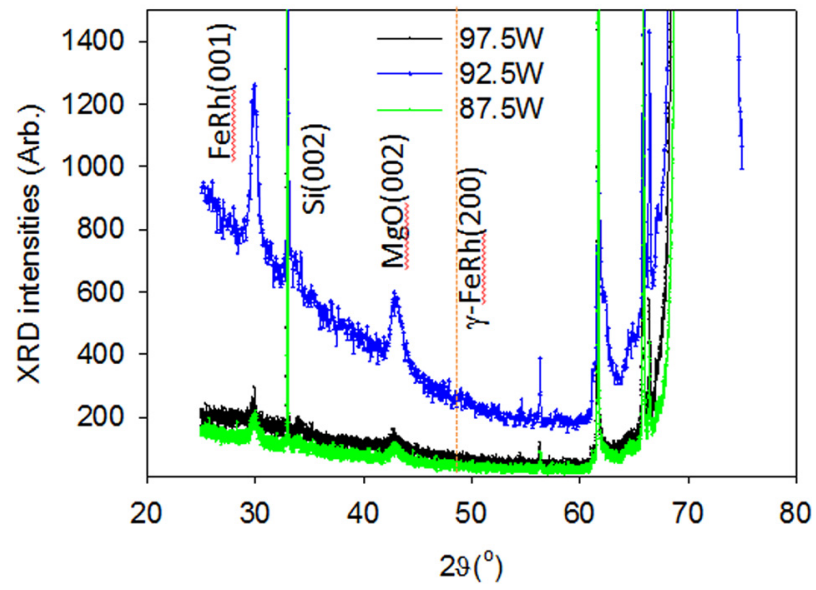

FIG. 3. XRD patterns of $10 \mathrm{~nm}$ thick B2 ordered FeRh films with different Rh contents. No $\gamma$ FeRh (200) peak is observed, indicating that there is no $\gamma$ FeRh phase formation even in the Rh-rich film.

calculations, ${ }^{10}$ which show that the AF phase is more stable than the FM phase at any thickness for the Fe-terminated film while the FM phase will be more stable only till 9 atomic layers for the Rh-terminated film. However, it should be pointed out that the calculations in Ref. 10 did not consider the interaction (FM exchange coupling) between FeRh grains in a real film. For Rh-terminated grains, the FM exchange coupling could be decoupled by Rh layer, thus stabilizing the AF arrangement within the grain. The exchange coupling induced FM stabilization could be demonstrated in the following result. As shown in Fig. 4, when a FM Fe layer was deposited on the FeRh layer before Ru capping layer at room temperature (to avoid the interdiffusion between the Fe layer and FeRh layer), the AF-FM transition in the film was completely destroyed. In contrast, for Ru capped FeRh films deposited subsequently, a clear AF-FM transition was observed. Similarly, Ding et al. ${ }^{15}$ also reported that the nearsurface/interfacial magnetism of FeRh may be modified by choice of capping layer. They found that even a nonmagnetic capping layer could induce FM stabilization in the FeRh film.

Our sputtering system is equipped with an inductively coupled plasma coil which allows for thin film deposition at

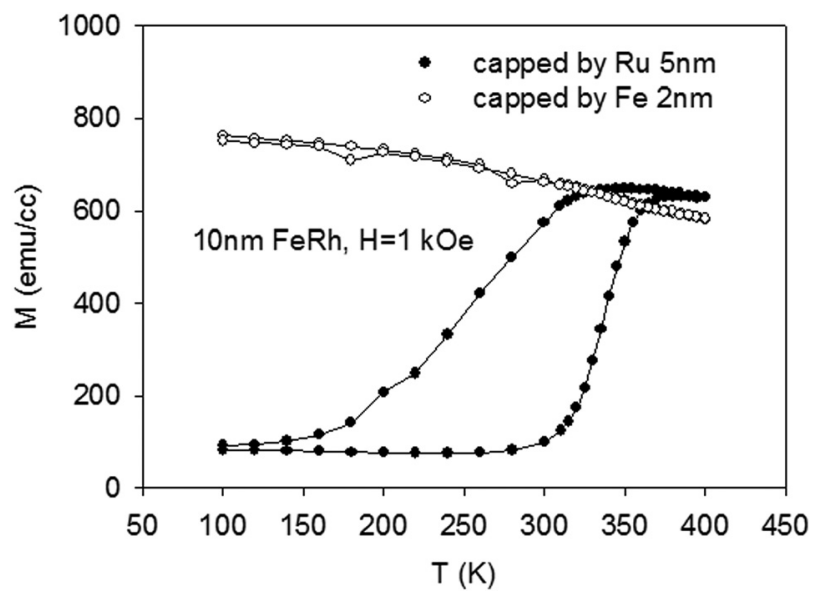

FIG. 4. M-T curves of $10 \mathrm{~nm}$ thick films capped by $\mathrm{Fe}(2 \mathrm{~nm})$ and $\mathrm{Ru}$ $(5 \mathrm{~nm})$. an extremely low pressure down to $0.057 \mathrm{~Pa}$. As discussed above, our ultrathin FeRh films exhibited lower residual magnetization and sharper transitions than reported so far in literature. To examine whether the good FeRh performance results from the low-pressure deposition or not, the magnetic properties of two samples deposited at a pressure of $0.105 \mathrm{~Pa}$ and $0.057 \mathrm{~Pa}$ were studied. At $0.105 \mathrm{~Pa}$, the composition was tuned and considered optimum when the lowest residual magnetization was achieved. At $0.057 \mathrm{~Pa}$, the composition was adjusted to be the same as the optimum composition achieved at $0.105 \mathrm{~Pa}$. Thus, the effects arising from composition difference are excluded when comparing the properties of these two samples. XPS measurements (not given here) confirmed that the two samples have exactly the same Fe and Rh content. Fig. 5 shows M-T curves measured at 1 kOe (the inset) and $50 \mathrm{kOe}$. At an applied field of $1 \mathrm{kOe}$, as shown in the inset of Fig. 5, the AF-FM transition is not yet completed as SQUID can only heat the sample up to $400 \mathrm{~K}$. An complete transition can be only observed at a high magnetic field. As shown in Fig. 5, when a field of $50 \mathrm{kOe}$ was applied, the transition is shifted below $400 \mathrm{~K}$. As a result, the larger magnetic transition was obtained and the magnetization at $400 \mathrm{~K}$ is much larger than that measured at $1 \mathrm{kOe}$. At a field of $50 \mathrm{kOe}$, not only was a small residual magnetization obtained, a sharp AF-FM transition was also observed for the sample deposited at $0.057 \mathrm{~Pa}$, while the sample deposited at higher pressure shows a broader transition with a larger residual magnetization. Such a small residual magnetization in the low-pressure deposited sample was further confirmed through magnetization curve (M-H) measurements. Figs. 6(a) and 6(b) show M-H curves measured at $100 \mathrm{~K}, 300 \mathrm{~K}$, and $400 \mathrm{~K}$ for the samples deposited at pressures of $0.105 \mathrm{~Pa}$ and $0.057 \mathrm{~Pa}$, respectively. For the FeRh film deposited at the pressure of $0.105 \mathrm{~Pa}$, obvious FM hysteresis loops were observed at a temperature down to $100 \mathrm{~K}$, while almost no hysteresis loop was detected at $300 \mathrm{~K}$ for the sample deposited at the pressure of $0.057 \mathrm{~Pa}$. However, it is also noted that at $100 \mathrm{~K}$ there is a small hysteresis loop for the low-pressure deposited sample, showing the existence of the FM phase. A careful examination of the M-T curve measured at $1 \mathrm{kOe}$ for

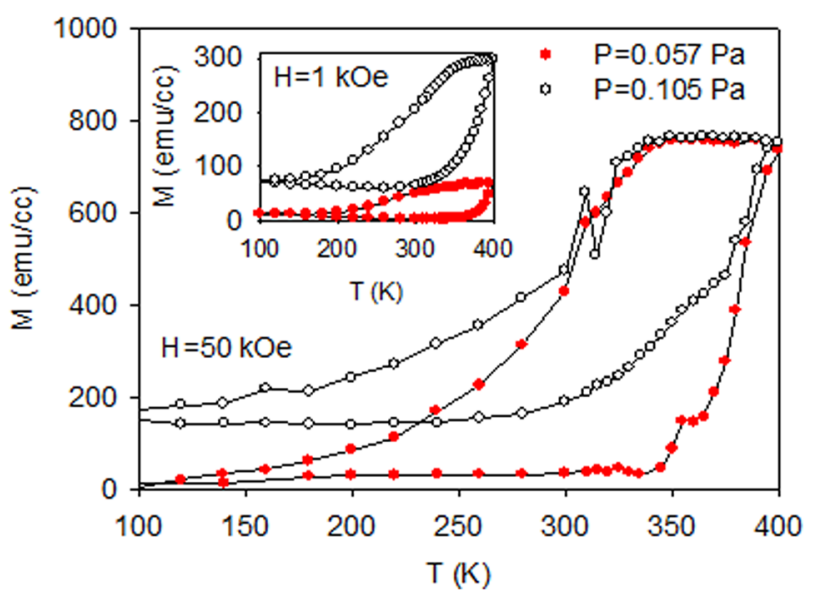

FIG. 5. M-T curves of $10 \mathrm{~nm}$ thick films deposited at different pressures measured at a field of $50 \mathrm{kOe}$. The inset gives the M-T curves measured at a field of $1 \mathrm{kOe}$. 

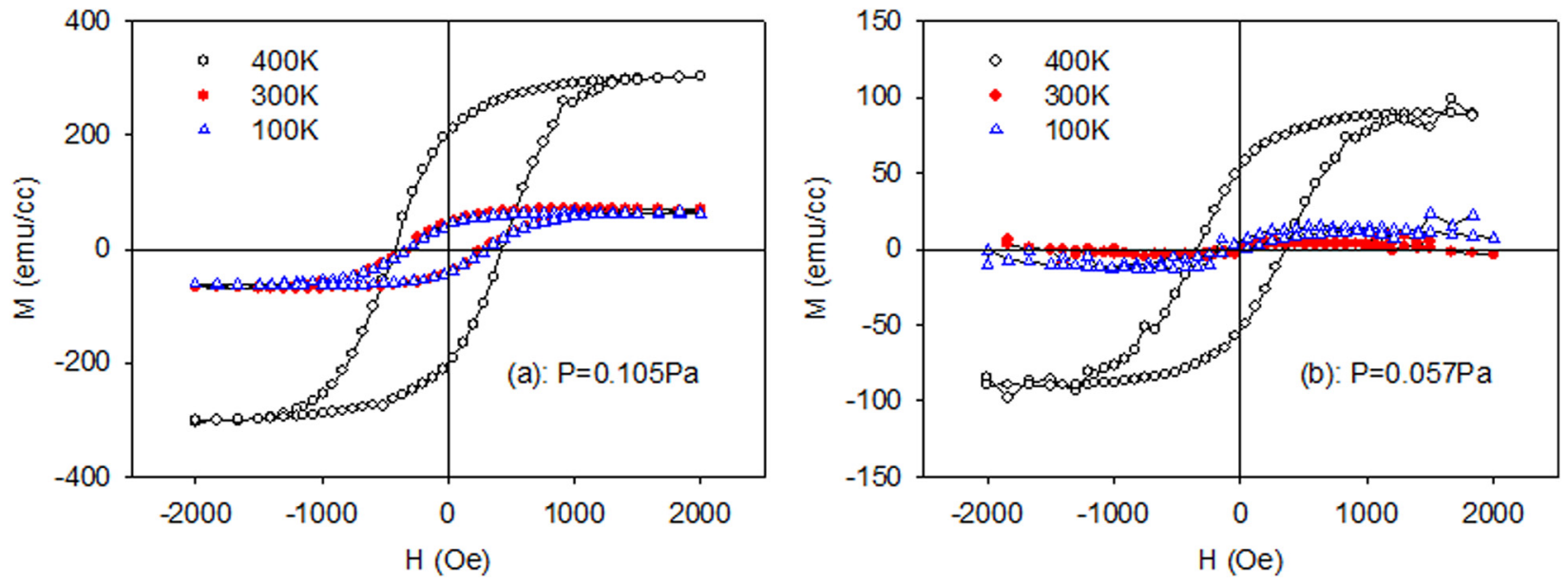

FIG. 6. Magnetization hysteresis loop of $10 \mathrm{~nm}$ thick FeRh films deposited at (a) $0.105 \mathrm{~Pa}$ and (b) $0.057 \mathrm{~Pa}$ measured at various temperatures.

the low-pressure sample shows that the magnetization decreases almost linearly from $13.5 \mathrm{emu} / \mathrm{cc}$ to $3 \mathrm{emu} / \mathrm{cc}$ as temperature increases from $100 \mathrm{~K}$ to $260 \mathrm{~K}$ before the onset of the AF-FM transition. Extrapolating this linear M-T curve to zero magnetization, we found that the Curie temperature of this FM phase is about $310 \mathrm{~K}$. As the composition was considered optimized, this FM phase could be ascribed to the interface/surface induced FM stabilization as predicted by Fan et al. ${ }^{9}$ and Lounis et al. ${ }^{10}$ Comparing the XRD results (not shown here) of these two samples, it was observed that the two samples have similar diffraction peaks in $\theta-2 \theta$ scans and the resulting order parameter is also similar $\left(\mathrm{I}_{001} /\right.$ $\mathrm{I}_{002}=0.94$ and 0.98 for $\mathrm{P}=0.057 \mathrm{~Pa}$ and $0.105 \mathrm{~Pa}$, respectively). Therefore, it is most likely that the smaller residual magnetization and the sharper transition observed for the film deposited at low pressure is from the more uniform composition distribution in the film which resulted from the higher energy of the sputtered adatoms arriving on the substrate due to the lower working pressure. In addition, as shown in Fig. 7, the grains in the film deposited at lowpressure pack more closely and are slightly larger in size. This could lead to an improvement in the strength of the magnetic coupling between the grains, which might be another reason for the sharper transition observed in Fig. 5.
It is noted that a certain amount of residual magnetization appears for all of our samples. Moreover, at a thickness of $10 \mathrm{~nm}$, the transition is much broader than that in thicker films. From the phase diagram, ${ }^{16}$ it can be seen that the transition temperature is expected to increase as Rh content increases within the $\alpha^{\prime \prime}$ phase region. Hence, a FeRh film having a continuous composition spread within the $\alpha^{\prime \prime}$ phase could correspondingly result in the onset of AF-FM transition occurring at a continuous spread of temperatures, thus possibly explaining the broadening of AF-FM transition. It is noted here that given the nature of the co-sputtering process, it is reasonable to expect some composition distribution in the deposited films. Other possible mechanisms responsible for the broadening include stress and defects. As pointed out by Kaneta et al., ${ }^{17}$ the AF structure would be destabilized when the amount of the site-exchange defect density exceeds the threshold of $0.8 \% / f$.u. In order to examine this effect, $\mathrm{MgO}(001)$ substrate was used to grow an ultrathin $(10 \mathrm{~nm})$ FeRh films to improve the crystallinity. For comparison, a $\mathrm{Si} / \mathrm{SiO}$ substrate was also loaded in the chamber in order to deposit the FeRh film simultaneously on both substrates. An $\mathrm{MgO}$ buffer layer was deposited on both substrates before the FeRh deposition. The M-T curves of the two samples are shown in Fig. 8. The magnetic transition is much sharper and

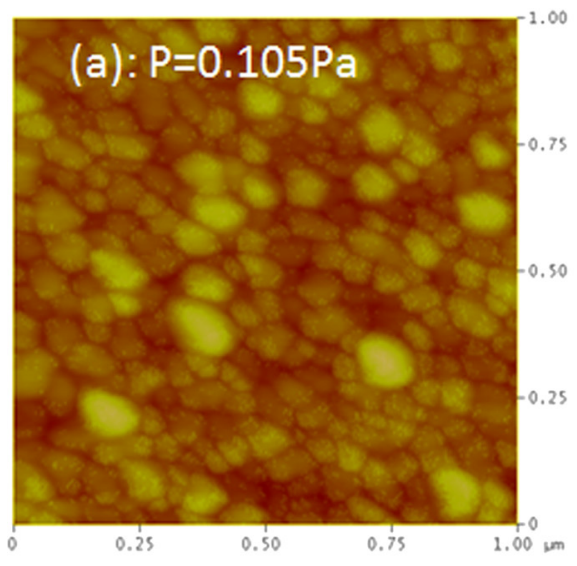

(a)

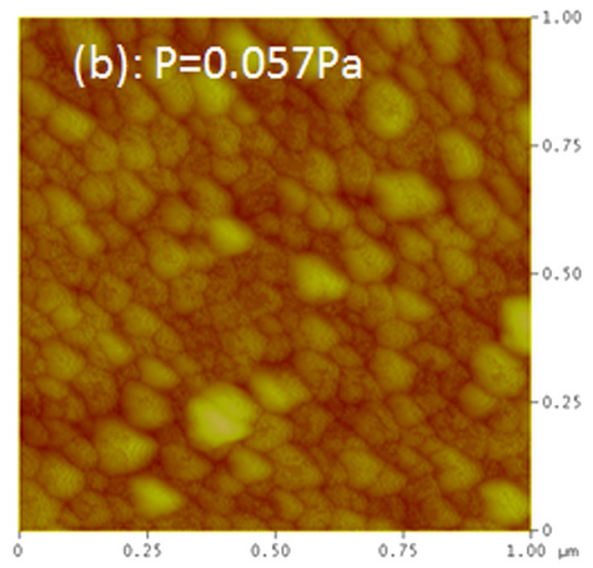

(b) 


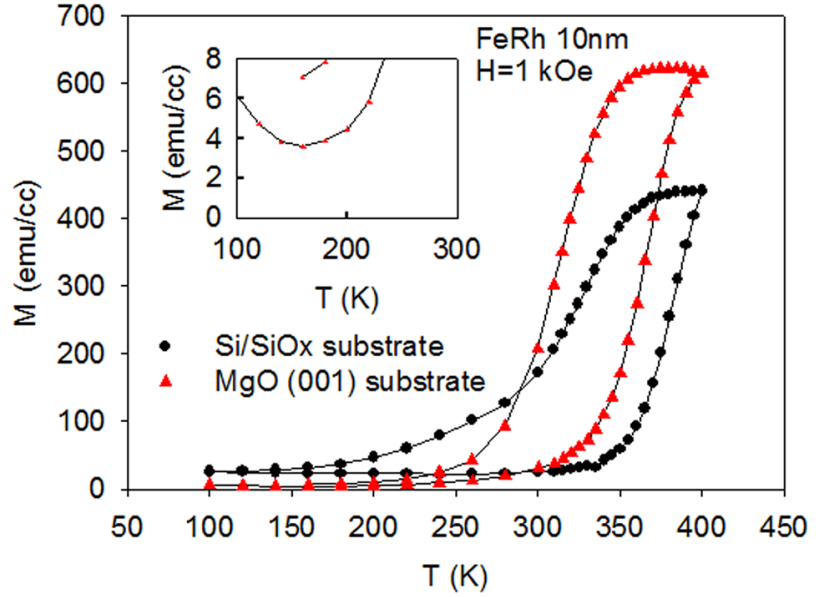

FIG. 8. M-T curves for $10 \mathrm{~nm}$ thick FeRh films deposited on $\mathrm{MgO}$ and $\mathrm{Si} / \mathrm{SiO}$ substrates. The inset shows the enlarged M-T curves at low temperatures.

the amplitude of magnetization transition was found to be larger for the film deposited on $\mathrm{MgO}$ substrate. The temperature hysteresis of the transition is $56 \mathrm{~K}$ and $60.6 \mathrm{~K}$ for the $\mathrm{MgO}$ and $\mathrm{Si}$ substrates, respectively. The hysteresis of the transition is much larger than that observed in thicker films, even for the $\mathrm{MgO}$ substrate. Such behavior was attributed to the decrease of the grain size in the ultrathin films. ${ }^{18}$ The stress and its distribution in the film could be another origin of the broader transition observed in the FeRh film grown on $\mathrm{Si}$ substrate. On the other hand, for the film grown on $\mathrm{MgO}$ substrate, the stress could be more uniform over the surface due to better crystalline structure of the $\mathrm{MgO}$ underlayer. In other words, the FeRh layer deposited on Si substrate might have a wider crystalline orientation distribution of FeRh lattice. That could cause a large distribution of the stress from the lattice mismatch between FeRh and $\mathrm{MgO}$ buffer layer, resulting in the broader transition. It is noted that for the FeRh film deposited on the $\mathrm{MgO}$ substrate, the residual magnetization is as small as $6 \mathrm{emu} / \mathrm{cc}$ at $100 \mathrm{~K}$, in contrast to $24 \mathrm{emu} / \mathrm{cc}$ for the film deposited on $\mathrm{Si} / \mathrm{SiO}$ wafer, implying the high stability of the AF phase at low temperatures for the former. Such low residual magnetization and sharp transition could be related to a high quality crystalline structure of the FeRh film. As shown in Fig. 9, the intensity of FeRh (001) XRD peak is much higher for the film deposited on the $\mathrm{MgO}$ substrate and this in addition to its narrow width indicates a much better crystalline structure in the FeRh film deposited on $\mathrm{MgO}$, since the two samples were deposited simultaneously and the thickness of the films is exactly the same. This suggests that the good magnetic properties could be achieved by improving the crystalline ordering of ultrathin FeRh films. However, as shown in the inset of Fig. 8, even the FeRh film on $\mathrm{MgO}$ substrate still exhibits some residual magnetization which decreases as temperature increases from $100 \mathrm{~K}$ to $160 \mathrm{~K}$, clearly demonstrating the existence of a FM phase. This FM phase might be attributed to the defect-induced FM stabilization. Further suppression of the residual magnetization could require a complete removal of the defects in the film.

As shown in Fig. 9, the FeRh (001) peak is shifted to the lower angle for the film deposited on $\mathrm{MgO}$ substrate,

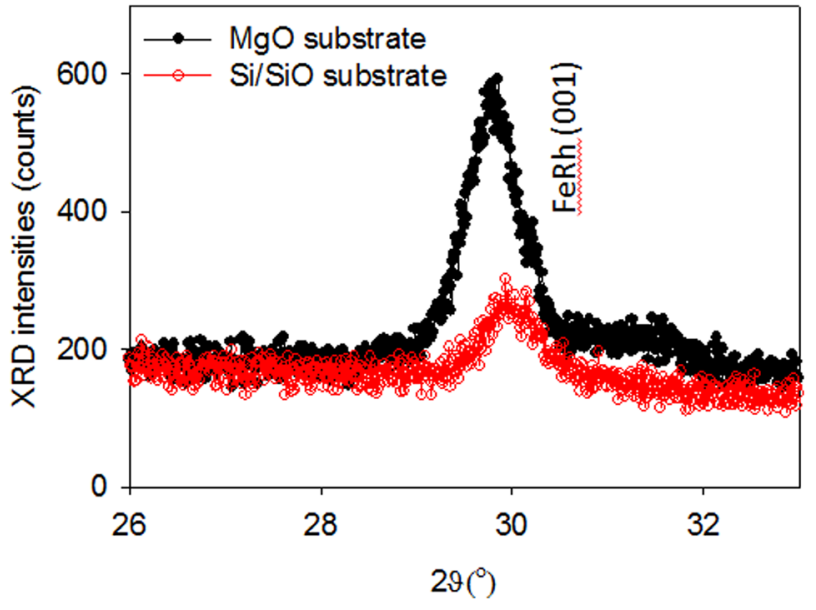

FIG. 9. XRD FeRh (001) peaks for samples deposited on $\mathrm{Si} / \mathrm{SiO}$ wafer and $\mathrm{MgO}$ substrate.

implying the expansion of the film along $\mathrm{c}$ axis. The lattice parameter c of FeRh films is estimated to be 2.984 and $2.999 \AA$ for $\mathrm{Si}$ and $\mathrm{MgO}$ substrates, respectively. This is similar to the result reported by Bordel et al., ${ }^{19}$ where FeRh grown on an ion-beam-assist-deposited $\mathrm{MgO}$ layer on a$\mathrm{SiO}_{\mathrm{x}} / \mathrm{Si}$ substrate was found to have a smaller c compared to FeRh grown on regular $\mathrm{MgO}$ substrates. The larger c parameter in FeRh grown on single crystalline $\mathrm{MgO}$ substrates is attributed to the in-plane contraction along the [100] and [010] axes due to the lattice mismatch between the FeRh film and the $\mathrm{MgO}$ substrate. This compressive stress would increase the magnetic transition temperature, ${ }^{12,13}$ which is contrary to the observation from Fig. 8. AFM images of the films (not given here) did not show much difference in the surface morphology of the two films. It is unclear why the transition temperature is lower for the FeRh film grown on $\mathrm{MgO}$ substrate. The large difference in the thermal expansion coefficients between $\mathrm{Si}\left(4.68 \times 10^{-6} / \mathrm{K}\right)$ and $\mathrm{MgO}$ $\left(13.5 \times 10^{-6} / \mathrm{K}\right)$ substrates might also have caused the variation in the transition properties. Since the thermal expansion coefficient of $\mathrm{MgO}$ substrate is larger, greater compressive stress might be produced in the FeRh film when the FeRh film deposited at $650{ }^{\circ} \mathrm{C}$ was cooled down to room temperature. The resultant effect could be such that the transition temperature of the FeRh film grown on $\mathrm{MgO}$ substrate is further increased. From the literature ${ }^{19,20}$ and the results shown in Fig. 1, the transition of the thicker $(>15 \mathrm{~nm})$ FeRh films is always shifted towards higher temperatures as the compressive stress in the film increases or is expected to increase, whereas for thinner films $(10 \mathrm{~nm})$, the transition always moved towards lower temperatures as reported in Refs. 6 and 21, and shown in Fig. 1. This suggests that the effect of the stress on the AF-FM transition could be different for thicker and thinner films. Because films typically relax stress by nucleating defects, a higher stress in thinner films will generally lead to more defects, which would stabilize the FM state at lower temperatures and thus lower the AF-FM transition temperature. A similar defect effect was reported by irradiation experiments using electron and heavy ions. ${ }^{22,23}$ While electron irradiation decreases the transition temperature by $3-18 \mathrm{~K}$ without changing the $\mathrm{B} 2$ crystalline structure, 
the ion irradiation induces the FM stabilization (no AF-FM transition was observed down to $5 \mathrm{~K}$ ).

\section{CONCLUSION}

In summary, systematic studies of ultrathin FeRh films were carried out to achieve good performance of magnetic transition properties. The sharp magnetic transitions and small residual magnetizations were achieved by optimizing the composition and depositing the films at an extremely low pressure. It is demonstrated that the residual magnetization of the ultrathin FeRh film are not only sensitive to the composition and its distribution, the crystalline ordering and granular structure, but also to the applied field and the interaction with the capping layer. Using $\mathrm{Ru}$ as the capping layer, residual magnetizations as small as $13.5 \mathrm{emu} / \mathrm{cc}$ and $6 \mathrm{emu} /$ $\mathrm{cc}$ at $100 \mathrm{~K}$ were observed for $10 \mathrm{~nm}$ thick films deposited on oxidized $\mathrm{Si}$ wafer and $\mathrm{MgO}(001)$ substrate, respectively. These results suggest that the FeRh films could be promising for practical applications in recording media and MRAM.

${ }^{1}$ E. Fullerton, S. Maat, and J. U. Thiele, U.S. patent 20050281081 (2005).

${ }^{2}$ J.-G. Zhu, Y. Luo, and X. Li, U.S. patent 7,826,258 (2010).

${ }^{3}$ J.-G. Zhu and D. E. Laughlin, U.S. patent, US2005/0281081A1 (2005).

${ }^{4}$ J. Thiele, S. Maat, and E. E. Fullerton, Appl. Phys. Lett. 82, 2859 (2003).

${ }^{5}$ D. Kande, S. Pisana, D. Weller, D. E. Laughlin, and J. G. Zhu, IEEE

Trans. Magn. 47, 3296 (2011).

${ }^{6}$ I. Suzuki, T. Koike, M. Itoh, T. Taniyama, and T. Sato, J. Appl. Phys. 105, 07E501 (2009).
${ }^{7}$ J. M. Lommel, J. Appl. Phys. 37, 1483 (1966).

${ }^{8}$ S. Hashi, S. Yanase, Y. Okazaki, and M. Inoue, IEEE Trans. Magn. 40, 2784 (2004).

${ }^{9}$ R. Fan, C. J. Kinane, T. R. Charlton, R. Dorner, M. Ali, M. A. de Vries, R. M. D. Brydson, C. H. Marrows, B. J. Hickey, D. A. Arena, B. K. Tanner, G. Nisbet, and S. Langridge, Phys. Rev. B 82, 184418 (2010).

${ }^{10}$ S. Lounis, M. Benakki, and C. Demangeat, Phys. Rev. B 67, 094432 (2003).

${ }^{11}$ J. W. Kim, P. J. Ryan, Y. Ding, L. H. Lewis, M. Ali, C. J. Kinane, B. J. Hickey, C. H. Marrows, and D. A. Arena, Appl. Phys. Lett. 95, 222515 (2009).

${ }^{12}$ L. I. Vinokurova, A. Vlasov, N. Kulikov, and M. Pardavi-Horváth, J. Magn. Magn. Mater. 25, 201 (1981).

${ }^{13}$ A. Hernando, J. M. Barandiarán, J. M. Rojo, and J. C. Gómez-Sal, J. Magn. Magn. Mater. 174, 181 (1997).

${ }^{14}$ J. van Driel, R. Coehoorn, and G. J. Strijkers, J. Appl. Phys. 85, 1026 (1999).

${ }^{15}$ Y. Ding, D. A. Arena, J. Dvorak, M. Ali, C. J. Kinane, C. H. Marrows, B. J. Hickey, and L. H. Lewis, J. Appl. Phys. 103, 07B515 (2008).

${ }^{16}$ T. B. Massalski, Binary Alloy Phase Diagrams (ASM International, Materials Park, OH, 1992), Vol. 2, p. 1760.

${ }^{17}$ Y. Kaneta, S. Ishino, Y. Chen, S. Iwata, and A. Iwase, Jpn. J. Appl. Phys., Part 1 50, 105803 (2011).

${ }^{18}$ Y. Ohtani and I. Hatakeyama, J. Magn. Magn. Mater. 131, 339 (1994).

${ }^{19}$ C. Bordel, J. Juraszek, David W. Cooke, C. Baldasseroni, S. Mankovsky, J. Minár, H. Ebert, S. Moyerman, E. E. Fullerton, and F. Hellman, Phys. Rev. Lett. 109, 117201 (2012).

${ }^{20}$ S. Maat, J.-U. Thiele, and E. E. Fullerton, Phys. Rev. B 72, 214432 (2005).

${ }^{21}$ J.-U. Thiele, S. Maat, J. L. Robertson, and E. E. Fullerton, IEEE Trans. Magn. 40, 2537 (2004).

${ }^{22}$ M. Fukuzumi, Y. Chimi, N. Ishikawa, F. Ono, S. Komatsu, and A. Iwase, Nucl. Instrum Methods B 230, 269 (2005).

${ }^{23}$ M. Fukuzumi, R. Taniguchi, S. Komatsu, F. Ono, A. Iwase, Mater. Res. Soc. Symp. Proc. 792, 393 (2004). 